\title{
Revue Gouvernance
}

Governance Review

\section{Une transition Canada Dry ? Les faux-semblants de la gouvernance des ressources naturelles au Canada}

\section{Amélie Dumarcher et Yann Fournis}

Volume 13, numéro 2, 2016

URI : https://id.erudit.org/iderudit/1039238ar

DOI : https://doi.org/10.7202/1039238ar

Aller au sommaire du numéro

\section{Éditeur(s)}

Centre d'études en gouvernance de l'Université d'Ottawa

ISSN

1912-0362 (numérique)

Découvrir la revue

Citer cet article

Dumarcher, A. \& Fournis, Y. (2016). Une transition Canada Dry ? Les

faux-semblants de la gouvernance des ressources naturelles au Canada. Revue Gouvernance / Governance Review, 13(2), 11-31.

https://doi.org/10.7202/1039238ar

\section{Résumé de l'article}

La transition économique est un enjeu majeur au Canada : initialement une colonie construite sur l'exploitation des ressources naturelles, il a depuis connu une évolution rapide, au point d'être considéré comme une puissance économique de premier plan. Toutefois, les évolutions récentes sont ambiguës et animent un débat lourd : pour certains, l'économie canadienne est appelée à dépasser le stade d'une économie centrée sur les ressources primaires pour s'inscrire dans l'économie du savoir ; pour d'autres, la période actuelle conduit plutôt à l'exacerbation de la vieille exploitation des ressources naturelles, mais sous des formes relativement nouvelles. Pour expliquer cette tension, nous examinons l'hypothèse du passage de l'économie canadienne d'un stade de maturation de l'exploitation traditionnelle (dit mature-staples) à un stade nouveau (dit " post-staples »). Cela permet de mettre à jour la variété et la profondeur des évolutions actuelles (économiques et productives, politiques et symboliques, sociales et territoriales) et de souligner qu'elles sont sans doute mieux abordées en termes de gouvernance et de jeu politique autour des ressources qu'en termes de "virage " objectif et structurel de l'économie politique des ressources. La transition de l'économie des ressources naturelles au Canada apparaît si hétérogène et inégale qu'elle pousse à questionner l'existence même d'un bouleversement structurel de ses bases. 


\title{
Une transition Canada Dry? Les faux-semblants de la gouvernance des ressources naturelles au Canada
}

\author{
Par Amélie Dumarcher et Yann Fournis ${ }^{i i}$
}

\section{Résumé}

La transition économique est un enjeu majeur au Canada : initialement une colonie construite sur l'exploitation des ressources naturelles, il a depuis connu une évolution rapide, au point d'être considéré comme une puissance économique de premier plan. Toutefois, les évolutions récentes sont ambiguës et animent un débat lourd : pour certains, l'économie canadienne est appelée à dépasser le stade d'une économie centrée sur les ressources primaires pour s'inscrire dans l'économie du savoir ; pour d'autres, la période actuelle conduit plutôt à l'exacerbation de la vieille exploitation des ressources naturelles, mais sous des formes relativement nouvelles. Pour expliquer cette tension, nous examinons l'hypothèse du passage de l'économie canadienne d'un stade de maturation de l'exploitation traditionnelle (dit mature-staples) à un stade nouveau (dit « post-staples »). Cela permet de mettre à jour la variété et la profondeur des évolutions actuelles (économiques et productives, politiques et symboliques, sociales et territoriales) et de souligner qu'elles sont sans doute mieux abordées en termes de gouvernance et de jeu politique autour des ressources qu'en termes de « virage » objectif et structurel de l'économie politique des ressources. La transition de l'économie des ressources naturelles au Canada apparaît si hétérogène et inégale qu'elle pousse à questionner l'existence même d'un bouleversement structurel de ses bases.

Mots-clés : Ressources naturelles, gouvernance, régimes de politique publique, transition économique, économie politique

\begin{abstract}
Economic transition is a major issue in Canada: initially a colony built on the exploitation of natural resources, it has since undergone a rapid evolution, to the point of being considered today a leading economic power. Recent developments, however, are ambiguous and lead to a challenging debate: for some, the Canadian economy is expected to move beyond a resource-based economy to the knowledge-based economy; for others, the current period rather leads to the exacerbation of the old exploitation of natural resources, but in partially renewed forms. To explain this tension, we examine the hypothesis of a transition in the Canadian economy, from a stage of maturation of the traditional exploitation (a mature-staples stage) to a new "post-staples" stage. This brings
\end{abstract}


light to the variety and depth of current developments (economic and productive, political and symbolic, social and territorial) and emphasizes that they are probably better addressed in terms of governance and political game around resources rather than as an objective and structural "shift" in the political economy of resources. The transition of the Canadian natural resource economy appears so heterogeneous and uneven that it raises questions about the very existence of a profound structural change of its bases.

Key words: Natural resources, governance, policy regimes, economic transition, political economy

\section{Introduction}

La transition de l'économie est si marquée au Canada que personne ne songerait plus à le qualifier de "pays sous-développé le plus riche du monde» iii. Après quelques décennies de développement, certains le considèrent désormais comme une «superpuissance » économique, tout au moins dans certains secteurs (comme l'énergie : Arès, 2014). Pour autant, cette affirmation reste encore récente et inquiète - ce dont témoignent aussi bien les débats sur la place des ressources naturelles dans l'économie nationale (avec ladite «maladie hollandaise » ou la «malédiction des ressources»), que les conflits autour des grands projets liés aux ressources (oléoducs, projets autour du gaz de schiste, parcs éoliens, etc.). Ces enjeux rappellent combien il est difficile d'échapper à son passé : alors que la grande passion économique de l'histoire canadienne a sans doute été de susciter une transition ou une maturation industrielle pour échapper à une trajectoire de colonie-ressource, ses plus récents succès semblent paradoxalement la renvoyer au statut de pays exportateur de matières premières (Stanford, 2008 ; Drache, 2009). À bien des égards, la transition économique du Canada ressemble sans doute à une maturation - mais, à bien y regarder, celle-ci concerne peut-être plus l'apparence que les structures profondes du développement canadien. Une transition Canada Dry ${ }^{1}$, en somme...

Bien que sa complexité décourage les analyses simplistes, cette trajectoire est suffisamment marquante pour que l'on s'interroge sur la configuration présente du Canada : comment interpréter aujourd'hui les dynamiques économiques, politiques et sociales d'une économie politique dont les fondements historiques extractivistes

\footnotetext{
1 Au cours des années 1980, en France, la marque Canada Dry faisait sa publicité sur le fait qu'elle partageait avec la bière un certain nombre de ressemblances, comme la couleur ou un pouvoir désaltérant, sans pour autant contenir d'alcool. Par extension, l'analogie au Canada Dry permet de souligner les situations où derrière une apparence commune, des phénomènes se révèlent finalement variés.
} 
semblent systématiquement remis en cause sans pour autant donner lieu à une recomposition d'ensemble? Comme les pays d'Amérique latine, qui abordent ces enjeux en termes originaux (l'extractivisme: Gudynas, 2013), le Canada a généré un corpus théorique original, autour de la théorie des staples dont les déclinaisons récentes tentent de saisir la complexité de la recomposition de l'économie du Canada, de ses provinces et territoires (Stanford, 2014).

L'hypothèse de l'émergence d'une "nouvelle économie», basée sur la connaissance et favorisée par la mondialisation contemporaine, a naturellement conduit à s'interroger sur la place que les ressources naturelles sont appelées à y occuper. Pour certains, l'économie canadienne est appelée naturellement à dépasser le stade d'une économie centrée sur les ressources primaires, pour s'inscrire pleinement dans la nouvelle économie globale de la connaissance ou du savoir ; d'autres remarquent cependant qu'il y a loin de la coupe aux lèvres et que, comme l'hypothèse commode d'une "nouvelle économie » reste discutée (Gadrey, 2003), la période actuelle semble bien plutôt conduire à une exacerbation, sous des formes nouvelles, de la vieille exploitation des ressources naturelles... Ces deux voies de sortie du modèle traditionnel d'exploitation des ressources naturelles semblent bien actuellement en jeu dans les conflits et débats qui les entourent. Pour en rendre compte, nous examinerons l'hypothèse du passage de l'économie canadienne d'un stade staples à un nouveau stade (dit "post-staples»), mais en soulignant que ses évolutions actuelles sont à la fois multiples et profondes (économiques et productives, politiques et symboliques, sociales et territoriales) et qu'elles sont sans doute mieux abordées en termes de gouvernance et de de jeu politique autour des ressources qu'en termes de « virage » objectif et structurel de l'économie politique des ressources.

Pour réévaluer cette dimension politique de l'économie, nous commencerons par poser quelques balises pour comprendre la portée du débat actuel staples / post-staples. Nous rappellerons d'abord que l'économie canadienne s'est historiquement construite sur l'exploitation des ressources premières, et que ce modèle de développement traditionnel subit actuellement des pressions nouvelles. Ces évolutions sont cependant loin d'être claires : si certains notent qu'elles reconduisent un même modèle de développement sous des formes de plus en plus intensives, d'autres postulent que ces tensions mènent plutôt à l'émergence d'un nouveau stade de développement (un stade post-staples, en rupture avec l'histoire). Nous finirons en suggérant que, observé sous l'angle de la gouvernance, il est difficile de proposer une conclusion tranchée: loin d'offrir le tableau d'une transition globale et cohérente, les différents secteurs économiques examinés connaissent des dynamiques hétérogènes et inégales, qui laissent apparaître à la fois des dynamiques de maturation d'un modèle classique et des dynamiques relevant d'une rupture certaine. 


\section{Développement économique canadien et rôle historique des ressources}

Le rôle du Canada sur la scène économique mondiale est historiquement défini par les activités liées aux ressources et leur exportation. Selon une définition communément admise, le terme staples peut être traduit par «matières premières" ou "principales ressources », en ce qu'il fait référence à "a raw or unfinished bulk commodity product that is sold in export markets» (Howlett, Brownsey, 2008: 4). Mais plus précisément, selon l'approche hétérodoxe d'H. Innis (par exemple, Innis, 1999), le concept de staples désigne un mode d'organisation sociale et économique de la ressource (y compris la production, le commerce et le peuplement) dont l'influence est si forte qu'il permet de définir l'ensemble d'un modèle de développement économique centré sur l'exploitation des ressources naturelles (Drache, 1982).

Car le développement économique canadien est marqué historiquement par une dépendance structurelle, sous deux dimensions. D'une part, l'économie canadienne s'est construite sur l'export de matières brutes sur les marchés européens, puis continentaux et mondiaux. En dépit d'évolutions majeures récemment (Drache, 2009), elle reste à ce jour marquée par une dépendance lourde aux exportations, par rapport à d'autres pays auxquels on serait tentés de la comparer (Barnes, 1996 ; Stanford, 2014). D'autre part, cette évolution ne s'est pas faite sans heurts, puisqu'il y a eu historiquement deux grands modèles d'exploitation liée aux ressources: l'entrepreneuriat localisé et le type «plantations» étrangères comme des branches de multinationales, qui ont des implications locales bien différentes. Le modèle entrepreneurial a atteint son pic au début du XXe siècle, avant que les grandes firmes du style " plantations » ne gagnent du terrain, et deviennent dominantes pendant la période fordiste (Barnes et coll., 2001). Cette double dépendance, aux marchés internationaux et aux entreprises étrangères, a mené Jenson (1989) à qualifier le fordisme canadien de "permeable fordism», au sens où le développement de l'économie canadienne est en permanence imprégnée par de fortes tendances internationales, qui exercent un impact majeur sur les dynamiques internes au Canada.

Traditionnellement (tout au moins jusqu'aux années 1990), l'exploitation et la mise en valeur des ressources peuvent être caractérisées comme suit. En termes de production, les ressources extraites étaient accessibles et abondantes, dans de grands espaces peu occupés. Elles étaient exploitées principalement par de grandes entreprises exogènes et exportées sur les marchés internationaux sous forme brute. Sous l'angle de la gouvernance et des arrangements institutionnels encadrant cette exploitation, un régime de politique publique stable et cohérent visait à soutenir un développement économique national et provincial basé sur les ressources, sur fond de consensus autour d'un 
paradigme extractiviste hégémonique. La gestion, de style hiérarchique, centralisé et corporatiste, excluait largement les acteurs sociaux et territoriaux. Localement, cela se traduisait par une dépendance aux grandes entreprises extérieures, et par une économie très peu diversifiée dont la forme la plus exacerbée reste la ville monoindustrielle. L'interdépendance économique entre les métropoles et leur hinterland était très marquée dans ce schéma (cf. Howlett, 2001 ; Hessing et coll., 2005 ; Fournis et coll., à paraître).

La théorie des staples se démarque donc de l'approche néoclassique et de son optimisme qui voudrait que le développement par les ressources soit une voie vers la diversification de l'économie (cf. Mackintosh, 1923, pour les travaux canadiens). Dans la suite des travaux d'Innis, Watkins insistera sur l'existence problématique d'un staple trap (piège des ressources) qui bloque les processus de diversification et contrarie un développement par étapes de type Rostow. Ce cadre d'analyse, avec quelques nuances et des avancées plus récentes, reste largement valide aujourd'hui pour penser l'économie canadienne (Watkins, 2007 ; Stanford, 2003, 2006, 2014). Il est aussi utilisé avec succès dans certaines provinces, comme en Colombie-Britannique, autour notamment des travaux de Barnes et Hayter en géographie économique, et d'Howlett, Hutton et Brownsey en économie politique. Il connaît également un regain d'intérêt dans la science régionale québécoise (Fournis, Fortin, 2013, 2014 a, 2014 b; Proulx, 2014). Il est aussi mobilisé dans les Maritimes (pour le Nouveau-Brunswick, voir Wallace, 2012), où une tradition d'économie politique critique, vive dans les années 1970 et 80, avait déjà permis d'aborder les effets pervers d'un développement économique lié aux ressources. Notons aussi qu'au fil des analyses, ce piège et cette dépendance ont été examinés à différents niveaux : l'économie canadienne dans son ensemble avec ses relations de dépendances internationales (Stanford, 2014), ou encore les villes monoindustrielles affectées par des dynamiques "cycloniques" (Barnes et coll., 2001), ou à l'échelle provinciale dans une articulation entre métropole et régions périphériques (notamment en ColombieBritannique, par exemple : Hutton, 1994, Markey et coll., 2008).

À ces questions initiales de la théorie des staples sur les blocages du développement se sont ajoutées des interrogations plus contemporaines sur la persistante des configurations traditionnelles: la crise du fordisme et la globalisation ont généré de nouvelles pressions qui ont complexifié le fonctionnement de ce modèle.

\section{Tensions et évolutions actuelles : Vers une rupture et un nouveau stade de développement post-staples?}

La crise du fordisme et l'ouverture accrue des frontières ont bousculé le modèle canonique des staples : les pressions et mutations dans l'exploitation des ressources ont 
introduit de nouveaux paramètres qui fragilisent ce modèle classique et ses politiques publiques (Hessing et coll., 2005). Pour éclairer ces tendances, plusieurs auteurs suggèrent l'existence d'un schéma séquentiel, spécifique aux économies des ressources, qui sert largement de base aux débats actuels. Selon l'hypothèse de Clapp (1998), les secteurs des ressources suivent une trajectoire de développement directement liée à l'épuisement des ressources facilement accessibles. C'est également l'hypothèse post-staples d'Hutton (2007), reprise par Howlett et Brownsey (2007 a, 2007 b, 2008).

Ces travaux distinguent quatre phases : après une période d'implantation rapide d'un modèle exploitant des ressources accessibles et abondantes s'ensuit une phase classique d'expansion et de consolidation du développement basé sur les ressources, puis une maturation rapide de l'exploitation (qui s'intensifie par la modernisation et l'organisation fordiste). Le dernier stade correspond à une grande accumulation de pressions depuis les années 1980 qui pourraient, selon l'hypothèse d'Hutton, mener à un épuisement définitif de l'exploitation des ressources et orienter l'économie canadienne dans une direction différente, avec l'émergence d'un nouveau stade de développement.

\subsection{Tensions et phénomène de maturation}

Avant d'examiner cette hypothèse d'un nouveau stade post-staples plus en détail, arrêtons-nous sur la phase de maturation qui le précèderait.

Le stade "classique», caractérisé par de nombreuses ressources accessibles et une production de type fordiste, serait suivi d'une "maturation» ayant les implications suivantes, selon les critères d'Hutton. D'une part, les problèmes de raréfaction et d'épuisement des stocks génèrent des pressions de groupes environnementaux et sociaux, des demandes sociales de conservation et d'amélioration de la gestion, et également la recherche de ressources de substitutions ou de nouveaux stocks par l'industrie. De plus, la compétition internationale porte à la fois sur le prix (avec de nouveaux pays exploitants plus compétitifs) et sur la qualité (avec d'autres pays producteurs en transition). L'impératif de flexibilité et d'ajustement rapide face à ces conditions mène à la restructuration de différents secteurs et industries, passant par une forte intensification capitalistique et technologique de l'exploitation, assez ambivalente (elle augmente la productivité et donne accès à de nouvelles ressources, mais diminue le nombre d'emplois associés). Le continentalisme reste central, mais réinséré dans un système économique élargi, global et plus instable. On note aussi une tendance à l'extension des chaînes de production en amont et en aval, depuis l'extraction basique vers la transformation et les services, ainsi qu'une tendance à la diversification dans les communautés avec une ouverture à d'autres activités non liées aux ressources. Bref, si ce stade mature-staples est toujours basé sur l'exploitation des ressources, l'économie y est 
plus diversifiée et ses dynamiques deviennent plus conflictuelles, sous l'impulsion de nouvelles forces.

La question centrale du débat actuel est donc de savoir si cette phase de maturation, avec l'accumulation d'instabilités et de nouveaux éléments, donne lieu à l'émergence d'un nouveau stade de développement où la place des ressources dans l'économie est révisée, et qui pourrait être qualifié de post-staples. Car les choses sont peu claires : même sans parier sur l'hypothèse maximaliste de l'émergence d'une "économie de la connaissance ${ }^{\text {iv }}$, il n'est pas évident de savoir si l'accumulation d'instabilités et de nouveaux éléments donne lieu à l'exacerbation des tensions traditionnelles au cœur du modèle staple de développement (hypothèse d'une maturation persistante) ou à l'émergence d'un nouveau stade de développement révisant plus radicalement la place des ressources dans l'économie (hypothèse qualifiée de post-staples).

\section{2 Émergence d'un nouveau stade de développement?}

Dans les deux dernières décennies, selon Hutton, des changements radicaux ont marginalisé structurellement l'économie des staples et leurs territoires, les régions ressources, accompagnant une transformation des représentations (en ce que l'extraction des ressources serait de plus en plus perçue par le public comme une activité résiduelle ou un vestige économique national). Toute la question est de savoir comment mesurer ces évolutions ; au côté des analyses économiques (Stanford, 2008 ; Cross, 2015), une piste s'ouvre pour les analyses politiques (Howlett et Brownsey, 2007a, 2007 b, 2008), qui se focaliseraient sur la gouvernance des ressources au sens large, c'est-à-dire sur les conditions du pilotage des restructurations diverses de l'économie politique des ressources naturelles.

L'hypothèse d'une rupture dans le modèle de développement n'est pas fondée uniquement sur un phénomène de restructuration industrielle et économique: elle implique aussi des changements dans les configurations sociopolitiques de l'exploitation des ressources, ainsi que la recomposition de leurs conditions territoriales (avec le repositionnement des villes) et sociales de possibilité (avec l'essor de mobilisations sociales) - toutes dimensions qui renvoient aux définitions actuelles de la gouvernance des ressources naturelles (Fournis et coll., à paraître). Plus précisément, trois types de contraintes et de dynamiques ont été identifiés par différents auteurs.

En premier lieu, la maturation industrielle et productive évoquée plus haut transforme les bases économiques du modèle d'exploitation des ressources, dans une logique d'intensification de la connaissance. Il y aurait un débordement (voire un déplacement) des activités et emplois liés aux ressources vers les secteurs secondaires et tertiaires associés, qui encadrent les activités primaires en amont et en aval. Sous l'effet de l'épuisement des stocks (qui pousse à la recherche de «nouvelles ressources»), ces 
tendances conduiraient à un virage vers une économie «du savoir», qui pénétreraient plus profondément le traditionnel développement lié aux ressources (qui conserveraient donc leur place importante). Ces tendances semblent à première vue assez bien illustrées dans les différents secteurs: organismes génétiquement modifiés dans l'agriculture (Moore, 2007), aquaculture pour le secteur de la pêche (Rayner, Howlett, 2007), virage éolien du modèle hydroélectrique (Netherton, 2007), regain des hydrocarbures par le gaz de schiste (Fortin, Fournis, 2013) ou encore des tentatives de diversification et de transformation plus poussée dans le secteur forestier.

En deuxième lieu, ces évolutions auraient des impacts majeurs sur la configuration spatiale de l'économie canadienne - ce qui a souvent conduit à considérer la croissance des villes et la métropolisation comme un indicateur de la diminution du rôle des ressources dans l'économie canadienne. Ainsi, selon Hutton, l'interdépendance centrepériphérie serait de moins en moins marquée, sur fond d'une disjonction entre des villes en croissance (qui se repositionnent facilement vers l'économie du savoir) et des régions et petites communautés en difficulté, parce que dépendant de secteurs de ressources structurellement fragiles. Cette déconnexion entre villes et régions jouerait aussi un rôle dans l'évolution des représentations urbaines vis-à-vis des ressources et des régionsressources. La tension est parfois forte entre l'exploitation traditionnelle et des demandes de conservation et de régulation forte issues de milieux urbains (l'exemple de la gestion forestière en Ontario : Chiasson et coll., 2011). Il y aurait un certain décalage dû à une déconnexion entre le mode de vie citadin, ponctué d'expériences récréatives reconnaissant une valeur iconique aux paysages canadiens considérés «typiques », et les réalités quotidiennes des communautés des régions, qui vivent des ressources. Ainsi, le fait que la société canadienne connaisse une montée des valeurs environnementales aurait des implications territoriales fortes. Si la demande sociale de conservation n'est pas un fait nouveau (datée vers 1880 pour la forêt, voir Blais, Boucher, 2013), elle s'accentue actuellement (pour le Québec: Batellier, Sauvé, 2011) et contribue sans doute à marginaliser le rôle des ressources et de leurs espaces dans l'économie nationale. Et ces évolutions touchent aussi les priorités et orientations de l'action publique : l'agenda gouvernemental fédéral se tournerait plus largement vers les problèmes des régions urbaines, sous l'impact des communautés épistémiques urbaines dont le poids politique contribuerait à privilégier les intérêts urbains au détriment des régions-ressources (Hutton, 2007, 2008). On peut certes argumenter, comme Markey et coll. (2008) que cette disjonction est aussi le fruit d'un virage de la gestion publique depuis les années 80 , où l'absence de vision stratégique globale et articulée des espaces centraux et régionsressources affecterait particulièrement les territoires périphériques. Mais dans les deux hypothèses, l'actuelle disjonction centre-périphéries est accompagnée par une moindre attention des gouvernements provinciaux pour les disparités régionales et les problèmes structurels des espaces périphériques. 
En troisième lieu, les équilibres politiques caractérisant la gouvernance des ressources naturelles au Canada seraient en voie d'épuisement - avec pour symbole l'essoufflement du paradigme ressourciste historiquement dominant. Plusieurs pistes vont dans le sens d'une rupture dans les configurations sociopolitiques et institutionnelles qui encadrent le développement des ressources : le virage vers un État «compétitif», la recomposition actuelle de l'action publique, et la montée des demandes sociales pour la conservation et la préservation de l'environnement. De nouvelles formes de gouvernance économique émergeraient d'abord, sur fond d'un abandon de politiques ambitieuses de développement régional au profit d'un soutien à la compétitivité et au potentiel des régions. L'hypothèse d'un virage post-staples est ainsi considérée par Wellstead (2007) à travers les formes d'État qui émergent suite à la crise de l'État keynésien. Il distingue deux formes d'État «compétitif» (Jessop, 1993), qui semblent actuellement coexister. Le premier est l'État «schumpeterien», typique du stade post-staples d'Hutton, qui vise à faciliter le passage à une économie du savoir et à de nouvelles formes de concurrence et de compétitivité. Le potentiel économique des secteurs lui-même y est révisé: les ressources premières sont de moins en moins reconnues comme leaders du développement économique, et le potentiel compétitif est mis de l'avant via un soutien aux activités à plus forte valeur ajoutée. La deuxième forme est celle de l'État « ricardien », qui fait appel à une autre forme de compétitivité et d'autres formes d'action publique, ciblant toujours les avantages comparatifs et le maintien des prix bas. Cette deuxième forme correspond plutôt à des logiques classiques d'exploitation des ressources premières et à la persistance d'un stade de maturation.

Toutefois, les transformations politiques sont bien plus larges: le nouveau stade de développement serait aussi marqué par une augmentation et une diversification des acteurs impliqués (ou souhaitant être impliqués) dans le débat. La complexification horizontale de la gouvernance produirait une pluralisation des acteurs : aux parties prenantes traditionnelles (l'État et la grande entreprise) viennent s'ajouter des groupes de citoyens, groupes écologistes, d'autres acteurs économiques plus nombreux avec la diversification. La complexification des formes de gouvernance se joue aussi dans une recomposition verticale de la gouvernance (Brenner, 1999a, 1999 b). Le débat sort de la scène nationale pour déborder par en haut et par en bas, avec une plus grande place des organisations internationales (comme l'OMC, les organismes de certification, ou encore Greenpeace) et supranationales (type ALENA), et l'entrée en jeu d'acteurs régionaux et territoriaux qui veulent leur mot à dire dans le débat (ce qu'illustrent notamment les débats en matière forestière: Hayter, 2003). Reste à savoir comment cette complexité nouvelle s'intègre dans les formes de gouvernance en transition, et à évaluer comment se produit concrètement l'ouverture du modèle traditionnel de gouvernance des ressources. 


\section{Vers une transition du modèle de développement lié aux ressources? Les apports des analyses par secteur}

Le débat actuel s'est donc éloigné des interrogations sur les formes de dépendance et le piège des ressources primaires pour évaluer dans quelle mesure ce blocage a été dépassé avec l'avènement d'une nouvelle période. La question est de savoir si la trajectoire classique se poursuit autour de logiques exacerbées et plus complexes, dans une maturation et une trajectoire de type mature-staples, ou si l'on assiste à l'émergence d'un stade nouveau où les ressources seraient moins centrales dans les structures de l'économie canadienne. À la suite des travaux sur les secteurs de ressources autour d'Howlett et Brownsey (2007 a, 2007 b, 2008), nous allons tenter d'estimer si les évolutions observables dans les différents secteurs (pêche, forêt, mines, éolien, hydrocarbures, et agriculture) permettent de conclure à une transition générale de l'économie politique des ressources canadiennes. Deux points clés semblent caractériser les transformations profondes actuelles: la crise qui affecte les différents secteurs n'est pas uniquement économique, mais largement multidimensionnelle, et les trajectoires de ces secteurs ne convergent ni vers une simple maturation ni vers une transition homogène vers un stade post-staples. Il semble donc que si une transition est amorcée, elle ne se comprendra pas en termes simples.

\subsection{Une crise multidimensionnelle et multiscalaire}

L'exploitation et la mise en valeur des ressources ne traversent pas seulement une crise économique : elles semblent aussi confrontées à une crise environnementale et politique, les trois étant largement imbriqués et relevant de différents niveaux de gestion.

Sur le plan environnemental, la diminution des stocks riches et accessibles affecte la plupart des secteurs. Les impacts environnementaux sur les écosystèmes inquiètent aussi, comme dans le cas des élevages de saumon (Rayner, Howlett, 2007), de l'ostréiculture (Lanteigne et coll., 2008), de certaines pratiques de pêche (pour la pêche aux pétoncles : Aubin, 2012), des mines (McAllister, 2007), dans le secteur forestier (avec l'impact des coupes à blanc; cf. par exemple Stefanick, 2001, ou l'essor du film «l'Erreur Boréale » (Desjardins, 1999) au Québec), pétrolier (Brake, Addo, 2014) et gazier (Fortin, Fournis, 2015) ou encore porcin (cf. Fournis et coll., à paraître). Les effets globaux sont aussi critiqués, comme en témoigne le débat sur les sables bitumineux et les émissions de GES (Pineault, Murray, 2016). Les réponses techniques, à la fois par les progrès techniques de l'industrie et les réglementations, semblent ne plus suffire à répondre aux nouveaux enjeux (pour les mines McAllister, 2007) ou aux inquiétudes des populations. Cependant, malgré l'importance des enjeux environnementaux, la crise actuelle ne porte pas seulement sur l'aspect écologique, mais sur une prise en compte de l'« environnement» des communautés au sens large, comme «ce qui les entoure » (Brunet, 1993), qui sousentendrait des enjeux non seulement écologiques, mais aussi sociaux et politiques. 
La crise est donc également une crise politique, qui semble se décliner en deux dimensions. Il s'agit d'une part d'une crise globale d'un mode de gestion en manque d'efficacité face à ces enjeux. On peut évoquer les effets pervers des accords internationaux de commerce, notamment continentaux: ils contribuent à institutionnaliser la fragilité structurelle des économies des ressources canadiennes et la position de faiblesse des producteurs dans certains secteurs comme l'agriculture (Skogstad, 2007). La gestion écosystémique des pêcheries est aussi entravée par les accords de commerce et les relations de pouvoir déséquilibrées (Hoogensen, 2007). La gouvernance forestière est aussi complexifiée par la fragmentation et l'imbrication des niveaux de gouvernance (Howlett, Rayner, 2010). D'autre part, c'est une crise de légitimité d'un mode de gestion historiquement bien ancré, descendant et centralisé, monopolisé par l'État et les industriels, autour de l'objectif d'extraction massive de matière abondante. Cette dimension de la crise est largement documentée dans le secteur forestier (voir notamment (Beckley, 1998, 2014 ; Blais, Chiasson, 2005 ; Chiasson, Leclerc, 2013). Le secteur minier révèle enfin un grand manque de confiance dans les relations, avec des acteurs nombreux, autour de demandes et enjeux variés (McAllister, 2007). Les revendications et mécontentements portent à la fois sur le partage de la ressource dans des configurations locales multiusages, comme dans la forêt; sur le partage des retombées autour de la question des rentes, comme dans le secteur minier; sur le partage du territoire comme dans le cas des revendications des Amérindiens, et enfin, de façon générale, sur le partage de la décision et l'inclusion des acteurs non traditionnels comme partie prenante.

\subsection{Des trajectoires ambivalentes}

S'il est possible de documenter solidement des facteurs généraux d'une crise généralisée des différents secteurs des ressources naturelles, il est bien plus difficile de démontrer son homogénéité : les trajectoires des différents secteurs ne convergent pas dans un processus de transition uniforme.

Sur le plan productif, l'intensification financière et technique tente de répondre à une géographie des ressources plus complexes à extraire, dont l'éloignement pose des questions de transport, ou à l'inverse où la proximité des implantations humaines soulève des problèmes de sécurité et des inquiétudes. Elle cherche aussi à répondre aux impératifs de rentabilité, y compris hors de l'industrie en intervenant sur le transport des ressources, ou sur celui des employés comme avec les migrations pendulaires («fly-in/fly-out»). Les réponses techniques se posent aussi face aux préoccupations environnementales, mais cela semble ne plus suffire, comme dans le secteur minier ou agricole. Notons aussi que si un virage vers le "savoir» se profile, c'est dans une diversification autour des ressources premières, vers le tertiaire et le secondaire: le cas de l'agriculture où les 
activités «beyond the farm gate » dominent est particulièrement éloquent (Skogstad, 2000). Dans la plupart des secteurs, certaines tendances post-staples semblent observables autour des activités de plus en plus tournées vers le savoir, mais ces activités ne signent pas toujours une véritable rupture avec les logiques classiques, et ne profilent pas de transition générale. En témoignent l'ambiguïté et l'éclatement des transformations structurelles en cours dans de nombreux secteurs: le passage de l'agricole à l'agroalimentaire vulnérabilise les producteurs (Skogstad, 2007), l'aquaculture est toujours marquée par la dépendance extérieure et loin de supplanter la pêche en termes de quantités (Rayner et Howlett, 2007), les technologies et expertises de pointe dans le secteur minier se situent principalement dans les métropoles (McAllister, 2007), l'exploitation des hydrocarbures se tourne vers le non-conventionnel (Clancy, 2007), ou encore le passage depuis l'extractif vers l'attractif dans la forêt ne suffit pas toujours à sortir les communautés des logiques classiques et d'une forme de « rentes » de l'extérieur (Howlett, Brownsey, 1996, Thorpe, Sandberg, 2007, Schmallegger, Carson, 2010). Ainsi, les différents secteurs indiquent certes des signes de renouveau et de dynamisme (montée en qualité, intensification technologique, etc.), mais présentent également de fortes tendances à la maturation, avec l'accentuation de logiques staples classiques (en particulier dépendance à l'extérieur et fragilité structurelle). Celles-ci peuvent même parfois être reconduites par des activités emblématiques d'un stade post-staples, comme le tourisme ou l'aquaculture.

Achevons par une brève description des dynamiques les plus marquantes de la gouvernance des ressources: sur fond de résistance des réseaux décisionnels traditionnellement dominants (Hessing et coll., 2005), les différents secteurs semblent converger vers une multiplication du nombre d'acteurs et une montée des contestations et des revendications. Même les projets considérés plus «verts » sont concernés, comme l'éolien (Fournis et coll., à paraître) ou l'ostréiculture (Lanteigne et coll., 2008). Outre la montée des préoccupations environnementales, cela suggère aussi l'importance croissante des construits sociaux qui entourent l'exploitation des ressources et l'existence d'enjeux forts de partage de la décision. La complexification des débats symboliques entourant les réseaux décisionnels (ce que Hessing et coll. (2005) considèrent comme la «policy community») du développement des ressources s'impose comme une constante, qui oblige à élargir le cercle des décideurs dans la plupart des secteurs (sur les secteurs forestier, éolien et porcin, cf. Fournis et coll., à paraître ; pour la forêt : Howlett, Rayner, 2001). Le bloc social hégémonique qui a longtemps prédominé s'érode, conduisant les gouvernements et l'industrie à reconnaître peu à peu une certaine légitimité aux différents acteurs, même si cette légitimité peine à se traduire par un réel pouvoir et une ouverture plus générale des institutions (par ex. pour la forêt: Chiasson, Andrew, Leclerc, 2008; Chiasson, Letendre, Voyer, 2013; pour la pêche: Marshall, 2001). Le 
développement ne peut plus se concevoir sans leur accorder une certaine attention, même si leur poids reste encore mineur.

\section{CONCLUSION}

Le débat actuel sur la transition de l'économie canadienne est vital pour tous ces pays qui, contrairement aux puissances dominantes traditionnelles, se sont historiquement insérés dans l'économie internationale en fonction de l'«avantage comparatif» que constituait leur dotation en ressources naturelles. À cet égard, la théorie des staples constitue une entrée de choix pour aborder les dynamiques de structuration et déstructuration des économies politiques centrées sur les ressources naturelles, parce que son analyse du modèle canadien d'exploitation des ressources prend au sérieux aussi bien ses réalités industrielles et productives que ses dynamiques politiques, sociales et territoriales. En particulier, l'analyse en termes de gouvernance nous paraît offrir un gain de finesse parce qu'elle permet d'aborder les facteurs macro-structurels dans une perspective institutionnaliste et contextualisée. Certes, ses enseignements sont moins percutants que ces grandes analyses épurées cherchant une «malédiction » (ou une «bénédiction ») structurelle des ressources. Car l'hypothèse d'une "grande » transition économique vers un stade post-staples s'impose finalement moins que la réalité d'une multiplicité de transitions sectorielles, complexes, irrégulières et multidimensionnelles, impliquant des échelles de gestion imbriquées, dont tous les éléments ne convergent pas vers une trajectoire cohérente. Il est aussi difficile de conclure à une rupture qu'à une continuité : les transformations lourdes de l'économie politique des ressources naturelles conduisent moins à leur marginalisation qu'à complexifier leur place qui, si elle reste centrale par certains aspects, reflète désormais des logiques plus complexes, certaines relevant de l'exacerbation de « vieilles » logiques, d'autres profilant certaines nouveautés. Si un nouveau stade est en émergence, il s'agirait plutôt d'un stade combinant diverses logiques, que d'une réelle rupture : le paradigme ressourciste dominant est contesté, s'érode, mais n'est pas remplacé par un nouveau paradigme cohérent malgré l'arrivée de nouveaux acteurs et de nouvelles idées (Hessing et coll., 2005). Il existe cependant des évolutions réelles, qui peuvent être résumées comme un double virage: le développement lié aux ressources semble ne plus pouvoir se faire sans une certaine ouverture à des acteurs plus nombreux et variés (même si cette ouverture reste largement symbolique); il semble également qu'il ne puisse plus s'envisager sans un minimum d'attention aux enjeux environnementaux. Et ces deux paramètres expliquent sans doute le désordre croissant animant les secteurs des ressources naturelles: de plus en plus d'acteurs tentent d'influencer les grands arrangements du modèle de développement traditionnel des ressources (en dépit d'un cadre qui privilégie largement les acteurs dominants, gouvernementaux et industriels). Ceci est suffisant pour contester l'efficacité 
et la légitimité du paradigme ressourciste traditionnel, mais la multiplication du nombre d'acteurs et l'arrivée des nouvelles idées autour du «développement durable» ne se traduisent en fait pas par l'émergence d'un nouveau paradigme uniforme et cohérent ne serait-ce que parce qu'il remettrait en cause les intérêts dominants.

L'une des leçons à retenir de ces analyses par secteurs serait peut-être qu'une approche balistique ou séquentielle du changement ne suffit pas à éclairer les recompositions du modèle canadien. Si le modèle linéaire semblait fructueux jusqu'à présent (Clapp, 1998), le stade actuel s'annonce plus complexe à saisir, comme en témoignent ces conclusions mitigées. Il semble nécessaire de sortir d'une lecture commode, parce que basée sur le postulat du changement (un «nouveau stade»). L'enjeu, bien réel, du rythme et de la portée du changement représente sans doute une véritable question empirique, et qui doit être traitée comme telle. C'est peut-être ce qu'apporte finalement la notion de gouvernance des ressources naturelles : elle reste ouverte à différentes interprétations (en termes de régimes sectoriels, en particulier) parce qu'elle est suffisamment large pour assembler différents facteurs de changement (structurels, agentiels, cognitifs) et évaluer leur convergence ou leur non-congruence (et tel semble être le cas ici). Pour examiner plus finement les processus actuels et leur complexité, la crise du modèle traditionnel pourrait être vue comme un état durable de tension et d'instabilité, plutôt que comme une bifurcation magistrale, devant générer assez rapidement soit un rééquilibrage (version mature staple), soit une rupture (version d'un nouveau stade post-staples)...

Il y aurait peut-être là une leçon à la fois analytique et politique sur le développement canadien. D'une part, sur le plan analytique, entre les analyses de «l'équilibre » et de «la ponctuation ", il y a peut-être l'espace pour une analyse des crises au sens de C. Hay, c'est-à-dire un «équilibre catastrophique » à la Gramsci, où les politiques de ressource connaissent une perte de légitimité sans précédent, sans jamais déboucher sur un moment décisif. Il en résulterait plutôt un « interrègne » inconfortable et incertain où le développement ne peut se faire qu'à la pièce, en fonction de rapports de force et de coercition ad hoc (Hay, 1995 : 19). Sur un plan politique, ces considérations conduisent aussi à relancer la vieille question canonique et pessimiste de $\mathrm{M}$. Watkins, qui réclamait une «histoire écologique des staples » parce qu'elle serait seule en mesure de rendre compte de « la rapacité de la variante canadienne de capitalisme » $(2007$, p. 220). L'un des grands mystères du Canada est peut-être cette impressionnante capacité à faire durer un développement insoutenable - y compris jusqu'à l'effondrement final (sur la morue dans l'Atlantique : Mather, 2013). Faire durer l'insoutenable : tout un programme... 


\section{Bibliographie}

Arès, Mathieu. 2014. «Le Canada superpuissance énergétique ? L'affaire Nexen et la crédibilité de la politique conservatrice envers la Chine » Canadian Foreign Policy Journal 20 (1) : 39-49.

Aubin, Maria. 2012. La voix (voie) de la mer: Impacts des changements politiques, sociaux et environnementaux sur les pratiques et les savoirs locaux des pêcheurs de homards de la région sud de la Gaspésie (Mémoire présenté dans le cadre du programme de maîtrise en Anthropologie pour l'obtention du grade de maître ès arts (M.A.)). Université Laval, $177 \mathrm{p}$.

Barnes, Trevor J. 1996. «Harold A. Innis: Local hero. » Logics of dislocation: models, metaphors, and meanings of economic space, Mappings. New York: Guilford Press, 206-228.

Barnes, Trevor J., Roger Hayter, et Elizabeth Hay. 2001. « Stormy weather: cyclones, Harold Innis, and Port Alberni, BC » Environment and Planning A 33 (12):2127-2148.

Batellier, Pierre et Lucie Sauvé. 2011. «La mobilisation des citoyens autour du gaz de schiste au Québec : les leçons à tirer » Gestion 36 :49-58.

Beckley, Thomas M. 1998. «Moving toward consensus-based forest management: A comparison of industrial, co-managed, community and small private forests in Canada » The forestry chronicle 74 (5):736-744.

Beckley, Thomas M. 2014. «Public Engagement, Planning, and Politics in the Forest Sector in New Brunswick, 1997-2014» Journal of New Brunswick Studies / Revue d'études sur le Nouveau-Brunswick $5: 41-65$.

Blais, René et Jacques L. Boucher. 2013. «Les temps des régimes forestiers au Québec » Guy Chiasson et Édith Leclerc (dirs.). La gouvernance locale des forêts publiques québécoises : une avenue de développement des régions périphériques?. Québec (Québec) : Presses de l’Université du Québec, 30-64.

Blais, René et Guy Chiasson. 2005. «L'écoumène forestier canadien : État, techniques et communautés - L'appropriation difficile du territoire » Canadian Journal of Regional Science 28 (3):487-512.

Brake, Wendy et Edward Addo. 2014. « Tourism and'Fracking'in Western

Newfoundland: Interests and Anxieties of Coastal Communities and Companies in the Context of Sustainable Tourism » International Journal of Marine Science 4 (2) :16-41.

Brenner, Neil. 1999a. «Beyond state-centrism? Space, territoriality and geographical scale in globalization studies » Theory and society 28 (1):39-78. 
Brenner, Neil. 1999b. « Globalisation as reterritorialisation : the re-scaling of urban governance in the European Union » Urban studies 36 (3) :431-451.

Brunet, Roger. 1993. «Environnement » Les mots de la géographie : dictionnaire critique 3e éd.. Montpellier : RECLUS ; Paris, 188.

Chiasson, Guy, Caroline Andrew et Édith Leclerc. 2008. « Territorialiser la gouvernance du développement : Réflexions à partir de deux territoires forestiers. » Canadian Journal of Regional Science 31 (3) :489-506.

Chiasson, Guy, Catalina Gonzalez et Édith Leclerc. 2011. «La gouvernance participative des forêts publiques : L'Ontario et le Québec, des chemins parallèles ? " Territoire en mouvement Revue de géographie et aménagement. Territory in movement Journal of geography and planning (11):19-32.

Chiasson, Guy et Édith Leclerc. 2013. La gouvernance locale des forêts publiques québécoises : une avenue de développement des régions périphériques ?. Québec (Québec) : Presses de l'Université du Québec.

Chiasson, Guy, Simon Letendre et Mathieu Voyer. 2013. «La gouvernance à la frontière : le cas du Conseil de gestion intégrée des forêts publiques du MadawaskaRestigouche » Revue gouvernance Printemps 2013. http://socialsciences.uottawa.ca/aruccura/fra/documents/chiasson_letendre_et_voyer_final.pdf (consulté le septembre 2, 2014).

Clancy, Peter. 2007. « Offshore Petroleum Politics: A Changing Frontier in a Global System » Policy and Society 26 (1):113-134.

Clapp, R. Alex. 1998. «The resource cycle in forestry and fishing » The Canadian Geographer/Le Géographe canadien 42 (2) :129-144.

Cross, Philip. 2015. Unearthing the Full Economic Impact of Canada's Natural Resources What are they? How important are they? Ottawa: Macdonald-Laurier Institute.

Desjardins, Richard. 1999. L'erreur boréale. Office National du Film du Canada.

Drache, Daniel. 1982. « Harold Innis and Canadian capitalist development » Canadian Journal of Political and Social Theory / Revue Canadienne de theorie politique et sociale 6 (1-2) $: 35-60$.

Drache, Daniel. 2009. «Canada's Resource Curse: Too Much of a Good Thing » Norteamérica 4 (1). http://revistas.unam.mx/index.php/nam/article/view/15430 (consulté le juin 22, 2016).

Fortin, Marie-José et Yann Fournis. 2013. «Facteurs pour une analyse intégrée de l'acceptabilité sociale selon une perspective de développement territorial : l'industrie du gaz de schiste au Québec (rapport complet) ». Document remis au Comité d'évaluation 
environnementale stratégique sur le gaz de schiste et au Ministère du Développement durable, de l'Environnement et de la Lutte contre les changements climatiques. Rimouski : UQAR/GRIDEQ-CRDT.

Fortin, Marie-José et Yann Fournis. 2015. « Une participation conflictuelle : la trajectoire territoriale des mobilisations contre le gaz de schiste au Québec » Participations 13 (3) :119-144.

Fournis, Yann et Marie-José Fortin. 2013. «La transition des régimes de ressources au prisme de la gouvernance : les secteurs forestier, porcin et éolien ». Étude S1-3 remise au Comité d'évaluation environnementale stratégique des gaz de schiste et au Ministère du Développement durable, de l'Environnement et de la Lutte contre les changements climatiques. Rimouski : UQAR/GRIDEQ-CRDT. [http://ees-gazdeschiste.gouv.qc.ca/wordpress/wpcontent/uploads/2013/12/Rapport-etude-S1-3_UQAR.pdf] (consulté le 26 février 2014).

Fournis, Yann et Marie-José Fortin. 2014a. «Canada and its Spaces: a Northern Extractivism in Transition? » Boletim Petróleo, Royalties e Região 44 (juin) :12-14.

Fournis, Yann et Marie-José Fortin. 2014 b. « Développement régional et politique des ressources au Québec. Les études régionales au GRIDEQ et le développement territorial de l'industrie éolienne » Virginia Etges et Silvio Cezar Arend (dirs.). Crises do capitalismo, estado e desenvolvimiento regional. Santa Cruz do Sul : EDUSNIC, 259-275.

Fournis, Yann, Marie-José Fortin, Marie-Claude Prémont et Pierre-Henri Bombenger. 2013. «Le sous-secteur éolien et la gouvernance du vent » Yann Fournis et Marie-José Fortin (dirs.). «La transition des régimes de ressources au prisme de la gouvernance : les secteurs forestier, porcin et éolien ». Étude S1-3 remise au Comité d'évaluation environnementale stratégique des gaz de schiste et au Ministère du Développement durable, de l'Environnement et de la Lutte contre les changements climatiques. Rimouski :

UQAR/GRIDEQ-CRDT, 53-104.

Fournis, Yann, Marie-José Fortin, Geneviève Brisson, Guy Chiasson et Marie-Claude Prémont. à paraître. Les régimes de ressources québécois au prisme de la gouvernance : les secteurs forestier, porcin et éolien. Québec: Presses de l'Université Laval.

Gadrey, Jean. 2003. «La Nouvelle Économie. Un mythe techno-libéral. » Diane-Gabrielle Tremblay et David Rolland (dirs.). La nouvelle économie : où ? Quoi ? Comment ? SainteFoy : PUQ, 11-26.

Gudynas, Eduardo. 2013. «Extracciones, extractivismos y extrahecciones. Un marco conceptual sobre la apropiación de recursos naturales » Observatorio del desarrollo 18 :18. Hay, Colin. 1995. « Rethinking crisis: Narratives of the New Right and constructions of crisis » Rethinking Marxism 8 (2) :60-76. 
Hayter, Roger. 2003. " "The war in the woods": Post-Fordist restructuring, globalization, and the contested remapping of British Columbia's forest economy » Annals of the Association of American Geographers 93 (3):706-729.

Hessing, Melody, Michael Howlett et Tracy Summerville. 2005. Canadian Natural Resource And Environmental Policy: Political Economy And Public Policy. Vancouver : UBC Press.

Hoogensen, Gunhild. 2007. « The Canadian Fisheries Industry: Retrospect and Prospect » Canadian Political Science Review 1 (1):42-56.

Howlett, Michael. 2001. Canadian Forest Policy: Adapting to Change. Toronto : University of Toronto Press.

Howlett, Michael et Keith Brownsey. 2007a. «Introduction to Special Issue on Canada's Staples Industries : Towards a Post-Staples State? »Canadian Political Science Review 1 (1) :1-7.

Howlett, Michael et Keith Brownsey. 2007b. «Introduction: Towards a Post-Staples State? » Policy and society 26 (1):1-7.

Howlett, Michael et Keith Brownsey. 2008. Canada's Resource Economy in Transition: The Past, Present, and Future of Canadian Staples Industries. Toronto : Emond Montgomery Publications.

Howlett, Michael et Kleith Brownsey. 1996. «From Timber to Tourism: The Political Economy of British Columbia » Politics, Policy and Government in British Columbia. Vancouver : UBC Press, 18-31.

Howlett, Michael et Jeremy Rayner. 2001. «The Business and Government Nexus: Principal Elements and Dynamics of the Canadian Forest Policy Regime. » Michael Howlett (dir.). Canadian forest policy: adapting to change, Studies in comparative political economy and public policy. Toronto : University of Toronto Press, 23-62.

Howlett, Michael et Jeremy Rayner. 2010. «Overcoming the challenges to integration: embracing complexity in forest policy design through multi-level governance ». IUFRO (International Union of Forestry Research Organizations) World Series 201028 :93-110.

Hutton, Thomas. 2007. « Contours of the Post-Staples State: The Reconstruction of Political Economy and Social Identity in 21st Century Canada » Policy and Society 26 (1) :9-29.

Hutton, Thomas. 2008. « The reconstruction of political economy and social identity in 21st-century Canada » Michael Howlett et Keith Brownsey (dirs.). Canada's Resource Economy in Transition: The past, present, and future of Canadian staples industries. 39-60. 
Hutton, Thomas A. 1994. Visions of a «post-staples » economy: Structural change and adjustment issues in British Columbia. Centre for Human Settlements, University of British Columbia.

Innis, Harold A. 1999. The fur trade in Canada : an introduction to Canadian economic history. Toronto, Ontario : University of Toronto Press.

Jenson, Jane. 1989. « 'Different'but not 'exceptional': Canada's permeable fordism » Canadian Review of Sociology/Revue canadienne de sociologie 26 (1):69-94.

Jessop, Bob. 1993. « Towards a Schumpeterian Workfare State? Preliminary remarks on post-Fordist political economy » Studies in political economy 40 (1) :7-39.

Lanteigne, Léon, Omer Chouinard et Andrew Boghen. 2008. «L'acceptabilité sociale des activités ostréicoles au Nouveau-Brunswick». http://asrdlf2008.uqar.ca/Papiers\%20en\%20ligne/LANTEIGNE-CHOUINARD.pdf (consulté le novembre 19, 2013).

Markey, Sean, Greg Halseth et Don Manson. 2008. «Challenging the inevitability of rural decline: Advancing the policy of place in northern British Columbia » Journal of Rural Studies 24 (4) :409-421.

Marshall, Joan. 2001. «Landlords, leaseholders \& sweat equity: changing property regimes in aquaculture » Marine Policy 25 (5) :335-352.

Mather, Charles. 2013. "From cod to shellfish and back again? The new resource geography and Newfoundland's fish economy » Applied Geography 45 :402-409.

McAllister, Mary Louise. 2007. «Shifting Foundations in a Mature Staples Industry: a Political Economic History of Canadian Mineral Policy » Canadian Political Science Review 1 (1) :73-90.

Moore, Elizabeth. 2007. «The new agriculture: Genetically-engineered food in Canada » Policy and Society 26 (1):31-48.

Netherton, Alex. 2007. «The Political Economy of Canadian Hydro-Electricity: Between Old "Provincial Hydros" and Neoliberal Regional Energy Regimes » Canadian Political Science Review 1 (1) :107-124.

Pineault, Éric et David Murray. 2016. Le piège Énergie-Est : sortir de l'impasse des sables bitumineux. Montréal : Écosociété.

Polèse, Mario et Richard Shearmur. 2002. La périphérie face à l'économie du savoir : La dynamique spatiale de l'économie canadienne et l'avenir des régions non métropolitaines du Québec et des provinces de l'Atlantique. Montréal : INRS Urbanisation, Culture et Société et l'Institut canadien de recherche sur le développement régional. 
Proulx, Marc-Urbain. 2014. «La périphérie nord face à un nouveau cycle » Organisations et territoires 23 (3) :25-35.

Rayner, Jeremy et Michael Howlett. 2007. « Caught in a Staples Vise: The Political Economy of Canadian Aquaculture » Policy and Society, Towards a Post-Staples Future? New Products and New Directions in Canada's Resource Industries 26 (1) :49-69. Schmallegger, Doris et Dean Carson. 2010. «Is tourism just another staple? A new perspective on tourism in remote regions » Current Issues in Tourism 13 (3) :201-221. Skogstad, Grace. 2007. « The Two Faces of Canadian Agriculture in a Post-Staples Economy » Canadian Political Science Review 1 (1) :26-41.

Stanford, Jim. 2003. «Back to the Hinterland » Facts from the Fringe (73).

Stanford, Jim. 2006. « A tale of two economies » The Globe and Mail (8 may 2006).

Stanford, Jim. 2008. «Staples, Deindustrialization, and Foreign Investment: Canada's Economic Journey Back to the Future »Studies in Political Economy 82 (1) :7-34.

Stanford, Jim. 2014. The Staple Theory @ 50. Reflections on the Lasting Significance of Mel Watkins' "A Staple Theory of Economic Growth ». Ottawa : Canadian Centre for Policy Alternatives.

Stefanick, Lorna. 2001. «Environmentalism and environmental actors in the Canadian forest sector » Michael Howlett (dir.). Canadian forest policy: Adapting to change University of Toronto Press. Toronto, 157-171.

Thorpe, Jocelyn et L. Anders Sandberg. 2007. « Knotty Tales: Canadian Staples and Post-Staples Forest Policy Narratives in an Era of Transition from Extractive to 'Attractive' Industries » Canadian Political Science Review 1 (1) :57-72.

Wallace, Lisa J. 2012. « An actor-network approach to Canadian forest research: The case of a New Brunswick policy debate » The Canadian Geographer/Le Géographe canadien 56 (3) :362-380.

Watkins, Mel. 2007. «Staples Redux » Studies in Political Economy 79 (79) :213-226.

Wellstead, Adam. 2007. « The (Post) Staples Economy and the (Post) Staples State in Historical Perspective » Canadian Political Science Review 1 (1) :8-25.

\footnotetext{
i Université du Québec à Rimouski, Centre de Recherche sur le Développement Territorial

ii Université du Québec à Rimouski, Centre de Recherche sur le Développement Territorial, Groupe de recherche interdisciplinaire sur le développement régional, de l'Est du Québec

iii Le propos est de Kari Polanyi Levitt, citée par Albo et Jenson 1997 : 223.
} 
iv Contrairement à d'autres (Polèse, Shearmur, 2002), l'hypothèse d'Hutton n'est pas celle d'une « nouvelle économie de la connaissance », qu'il considère lui-même comme exagérant l'importance des activités liées au savoir et masquant l'importance que les ressources continuent d'avoir. Dans ce nouveau stade, les ressources occuperaient encore une place importante, mais différente : les efforts tournés vers le savoir s'articulent largement autour de la base des ressources premières, par exemple vers la transformation plus poussée de ces ressources dans le secteur secondaire, ou vers les services et technologies nouvelles associés à l'exploitation. 